\title{
Principios de valoración económica fundamentados en la teoría financiera: estudio en Pyme transportistas Maracaibo-Venezuela ${ }^{1}$ \\ Principles of economic valuation fundamentals in financial theory: study in Pyme transporters Maracaibo-Venezuela
}

DOI: http://dx.doi.org/10.17981/econcuc.38.1.07

\author{
Lilia Caicedo ${ }^{2}$ \\ Franklin Angulo Rangel ${ }^{3}$
}

\section{Resumen}

\begin{abstract}
El objetivo del artículo es analizar los principios de valoración económica fundamentados en la teoría financiera aplicados en las pequeñas y medianas empresas (pyme) de Maracaibo, Venezuela. Para desarrollarlo, se diseñó una investigación descriptivaanalítica de campo. La población estuvo constituida por 15 pymes del sector transporte de carga pesada ubicadas en Maracaibo. La información obtenida fue contrastada con la opinión de analistas del sector financiero del contexto en estudio. Se estableció que las empresas observadas utilizan técnicas de valoración de tipo histórico, basadas en los principios de liquidez y rendimiento. Los analistas y consultores indicaron que la información financiera suministrada por las pymes no resulta adecuada para aplicar métodos dinámicos basados en los principios financieros y que, generalmente, sus dictámenes no coinciden con las expectativas de los empresarios. Se concluye que las pymes transportistas aplican modelos de valoración financiera no apropiados en el entorno venezolano y no utilizan métodos para evaluar el riesgo.
\end{abstract}

Palabras clave: principios financieros; gestión financiera; técnicas de valoración económica; Pyme; sector transporte

Recibido: 3/2/2017 Devuelto para revisión: 12/5/17 Aceptado: 28/5/2017.

\begin{abstract}
${ }^{1}$ Articulo derivado del Proyecto de investigación: Principios de valoración fundamentados en la teoría financiera aplicados en empresas venezolanas del municipio Maracaibo, registrado en el Consejo de Desarrollo Científico y Humanístico de la Universidad del Zulia. Venezuela

${ }^{2}$ Lcda. en Administración; MgS en Educación. Investigadora responsable. Profesora de la Universidad del Zulia - Venezuela. liliacaic@gmail.com

${ }^{3}$ Contador Público; MgS en Gerencia Financiera. Co-investigador principal. Profesor de la Universidad de la Guajira Colombia. franklinangulorangel@uniguajira.edu.co
\end{abstract}

- The author; licensee Universidad de la Costa - CUC. Económicas CUC vol. 38 no. 1, pp. 147-164. Enero - Junio, 2017 Barranquilla. ISSN 0120-3932 Impreso, ISSN 2382-3860 Online

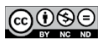




\begin{abstract}
The objective of this paper is to analyze the principles of economic valuation based on financial theory applied in the small and medium enterprises of Maracaibo- Venezuela. To develop it, a descriptive-analytical, field research was designed. The population was constituted by 15 SMEs of the sector of heavy load transport, located in Maracaibo. The information obtained was contrasted with the opinion of analysts of the financial sector of the study context. It was established that the observed companies use valuation techniques of a historical nature, based on the principles of liquidity and performance. Analysts and consultants indicated that the financial information provided by SMEs is not adequate to apply dynamic methods based on financial principles and that their opinions generally do not match the expectations of entrepreneurs. It is concluded that SME carriers apply non-appropriate financial valuation models in the Venezuelan environment and do not use methods to assess risk.
\end{abstract}

Keywords: financial principles, financial management, valuation methods and techniques, transport sector.

\title{
Introducción
}

La interrelación entre las decisiones de financiamiento, inversión y el riesgo, ha sido tema de discusión y análisis por parte de los integrantes de la comunidad financiera. En este sentido, destacan aportes valiosos sobre modelos de valoración de activos, riesgo, efectos del financiamiento, entre otros, que apoyan el proceso de toma de decisiones. Sin embargo, estos modelos tienden a focalizarse en recursos materiales y permiten una valoración de los resultados expresados como rendimientos sobre los activos, toda vez, que los cálculos se realizan sobre las inversiones tangibles como edificaciones, maquinarias, y su capacidad de generación de ingresos futuros. 
Se plantean entonces, inquietudes sobre la forma adecuada de valorar el desempeño, cómo medir la eficiencia de la gerencia financiera, cuánto vale realmente la empresa, por lo tanto, plantear sobre este particular, tiene gran sentido y relevancia para el sector empresarial y los profesionales del área financiera. En la actualidad, lograr la supervivencia de una organización en un medio económico en el que predomina la incertidumbre, la gerencia financiera debe ser capaz de realizar mejoras continuas, seguimiento a sus funciones claves, asumir los avances de la internacionalización, los convenios de integración, tratados de comercio, procesos que contribuyen a complejizar y dinamizar el ambiente organizacional.

Esto último, resulta muy pertinente cuando se trata de valorar el desempeño financiero de pequeñas y medianas empresas transportistas ubicadas en el contexto venezolano, caracterizado en los últimos años por un alto grado de incertidumbre relacionados con las disposiciones fiscales, financieras, laborales, económicas que rigen el comportamiento empresarial en Venezuela.

En este sentido, el presente artículo tiene por objetivo analizar los principios de valoración económica fundamentados en la teoría financiera aplicados por las pequeñas y medianas empresas transportistas de carga pesada, ubicadas en el municipio Maracaibo.

\section{Principios de valoración financiera}

El proceso financiero comprende una serie de actividades coordinadas como: planificar y ejecutar planes, sincronizar flujos monetarios, minimizar costos, establecer controles, incrementar la tasa de utilización de recursos, los niveles de efectividad, entre otras. Estas actividades y sus resultados son objeto de valoración mediante la aplicación de herramientas cuantitativas que permiten relacionar los logros alcanzados con el volumen de recursos utilizados.

Al respecto Stewart (2000), considera que la valoración es la esencia de la planificación y que sin un método para medir los efectos de las estrategias financieras en el valor de una empresa, la toma de decisiones puede ser desacertada. En este sentido, Fernández (2002), señala que la comprensión de los mecanismos de valoración de empresas es indispensable para toda persona involucrada en la función financiera no sólo por la necesidad de evaluar 
las decisiones de inversión, sino también para valorar el desempeño de la empresa y de sus unidades de negocio mediante la identificación de las fuentes de creación y destrucción de valor económico en la empresa.

Igualmente, Copeland, Koller \& Murrin (2004), detallan que la movilidad del capital y la globalización han influido en la importancia actual de la valoración como herramienta para tomar decisiones de inversión y la gestión basada en el valor, en esta línea de ideas, se ubican Bodie, Kane, \& Marcus, (2004), quienes enfatizan la necesidad de aplicar modelos de valoración para determinar la potencialidad de las inversiones en activos financieros.

Es evidente entonces la necesidad del gerente financiero de valorar sus decisiones aplicando los mecanismos, herramientas o modelos a que hacen referencia los autores mencionados, los cuales se basan en principios fundamentados en la teoría financiera, que para efectos de esta investigación pueden definirse como guías o lineamientos que orientan la valoración de las decisiones y que permiten comprender las transacciones y la toma de decisiones en el área financiera.

Según Macareñas (2007), estos principios pueden agruparse considerando los siguientes aspectos:

- Ambiente económico-competitivo: hace referencia a las preferencias del inversionista, bidireccionalidad de las operaciones financieras, expectativas y tendencias del mercado.

- Valor y eficiencia económica: en esta categoría se agrupan elementos referidos a la generación de ventajas competitivas y creación de valor.

- Transacciones financieras: alude a elementos y consideraciones presentes en una operación financiera, como el valor temporal del dinero, la diversificación de la inversión, la comparación riesgo-rendimiento y comportamiento del mercado.

Igualmente, Pérez (2009), destaca diez principios, pero no los agrupa en categoría alguna, sin embargo para fines de este trabajo, se organizan de la siguiente manera:

- Propósito: plantean lineamientos que orientan la toma de decisiones financieras e incluyen maximización del capital invertido, diversificación, desplazamiento de los recursos, financiamiento apropiado, apalancamiento financiero y ciclo del negocio. 
- Valoración: comprende factores que permiten evaluar decisiones, tales como, dilema riesgo-rendimiento, valor temporal del dinero, preferencia por la liquidez y costo de oportunidad.

Como puede observarse, los principios de valoración se desprenden de la teoría financiera y son el resultado de estudios y observaciones del desempeño de las empresas y mercados financieros desde el siglo XX hasta la actualidad, entre los que destacan, Black \& Scholes (1973), para fijación de precios de opciones, Merton (1973), precios de acciones, Jensen (1976), teoría de agencias, es decir, que la investigación en finanzas, ha consolidado como área de estudio la medición de valor de los activos, tomando en consideración factores internos como explica Jensen y factores externos como la dinámica de los mercados financieros. (Berk, DeMarzo \& Harford, 2010)

Atendiendo a los planteamientos expuestos, se puede señalar que los principios de valoración financiera permiten formular técnicas cuantitativas y parámetros cualitativos para evaluar decisiones financieras considerando elementos como el rendimiento, el riesgo, el valor del dinero en el tiempo, la liquidez y el costo de oportunidad asociados a las actividades de financiamiento e inversión de una organización. Al respecto, Garay \& González (2015), explican diferentes técnicas para valorar el desempeño de una organización, evaluar alternativas de inversión, medir el riesgo financiero, todas ellas basadas en los principios que a continuación se describen:

a. Rendimiento: es uno de los aspectos más analizados en una inversión, empresa en marcha, proyecto y de acuerdo con Eccles (2003), los métodos de medición del rendimiento son los más perfeccionados y arraigados en la teoría e instituciones financieras y son objeto de múltiples validaciones en empresas de cualquier tamaño y sector de la economía.

Generalmente las medidas de rendimiento pretenden estimar la contribución de la inversión u operaciones de la empresa al patrimonio de los inversionistas, se calcula de manera porcentual como una tasa de rendimiento, como incremento monetario sobre la inversión o como el número de veces que se recupera la inversión. Estos métodos son de carácter histórico o estático pues miden la rentabilidad para un periodo determinado.

Otro método utilizado por los analistas es la tasa interna de rendimiento que permite estimar la rentabilidad resultante de una serie de ingresos esperados, valor actual de los flujos, procedimientos sustentados por el principio del valor temporal del dinero. 
b. Riesgo: toda decisión financiera involucra un riesgo potencial, en este sentido puede citarse a Gitman \& Zutter (2012), quienes definen el riesgo financiero como "la incertidumbre en torno al rendimiento que ganará una inversión o, en un sentido más formal, el grado de variación de los rendimientos relacionados con un activo especifico" (p. 287)

Quiere decir entonces, que el inversionista está consciente de la posibilidad de obtener resultados no deseados y los analistas utilizan técnicas para estimar el impacto del riesgo y la variabilidad de los retornos esperados. La teoría financiera asume que los inversionistas son adversos al riesgo y por lo tanto elegirán opciones de inversión con menor grado de riesgo, así como también exigirán mayores niveles de rendimiento para las inversiones con mayor riesgo.

c. Valor: el indicador más adecuado para medir el éxito de la empresa se relaciona con su capacidad para crear valor para el accionista. La práctica financiera ofrece una serie de parámetros que pretenden evaluar la calidad de la gestión y que, con mayor o menor intensidad, se relacionan con la creación de valor para el accionista. El mensaje básico que transmite la medición del valor agregado a la empresa es que para añadir valor al capital invertido por los accionistas es necesario obtener, por lo menos, el costo de los recursos utilizados, y esto es una idea que está en el centro de la teoría moderna de las finanzas empresariales.

Siguiendo la definición aportada por Stewart (2000), el valor económico agregado puede calcularse como una medida de beneficio residual que se obtiene al restar el costo de los recursos utilizados de los beneficios operativos netos generados por la empresa y puede realizarse sobre valores históricos o proyectados.

d. Liquidez: la rapidez con la cual se recupera una inversión es un factor que es objeto de medición debido a la necesidad de las empresas de mantener efectivo bien sea para cubrir sus obligaciones a corto plazo o para aprovechar oportunidades de inversión. (Gitman \& Zutter, 2012). Es por ello que a juicio de Ibarra (2001), los objetivos de la medición de la liquidez están referidos a verificar la calidad de los activos circulantes, las reservas de fondos líquidos, solvencia a corto plazo, capacidad de generación de fondos. Igualmente señala que la valoración de la liquidez debe ser dinámica, utilizando para ello las estimaciones de flujos de fondos o flujos de caja, de manera que el responsable de la valoración pueda estudiar la capacidad de pago de la empresa y recuperación de las inversiones. 
Asimismo, al valorar oportunidades de inversión que presentan rendimientos similares, podrá utilizarse la liquidez como parámetro de decisión, es decir, seleccionar la alternativa con mayor rapidez de recuperación, sobre todo en economías inflacionarias.

e. Costo de oportunidad: generalmente el costo de oportunidad se interpreta como el beneficio que se podría obtener en una inversión distinta a la actividad ordinaria de la empresa. Garay \& González (2015), explican que el costo de oportunidad es la rentabilidad generada por una inversión alterna y representa el rendimiento máximo que deja de percibirse si los fondos se invierten en un proyecto determinado. Igualmente, Bodie \& Merton (2003), expresan que el costo de oportunidad debe ser utilizado como tasa de descuento para valorar los proyectos de la empresa, ya que es el rendimiento que obtendría en otra inversión diferente a la que se está evaluando.

Sobre este último señalamiento, cabe mencionar que los métodos de valoración utilizan generalmente el costo del financiamiento total (deuda y patrimonio), o el costo de la deuda, para determinar la contribución económica de las inversiones, pero al utilizar el costo de oportunidad, el analista estaría verificando si el proyecto es rentable de acuerdo a la tasa de capitalización del mercado.

\section{Contexto de las pyme transportistas del municipio Maracaibo}

Las empresas de transporte de carácter familiar ubicadas en el municipio Maracaibo, han sido creadas a partir del esfuerzo personal de sus fundadores, quienes comenzaron como trabajadores (conductores y gerentes en su mayoría), de otras empresas que tenían presencia a nivel nacional a través de operadoras y sucursales, ubicadas en las principales ciudades del País. Sin embargo, la demanda local, cada vez más creciente impulsó la creación de pequeñas organizaciones de transporte que se limitaban a realizar fletes locales o regionales y en ocasiones, asumían la figura de transportistas afiliados.

Desde su creación hasta los actuales momentos, estas organizaciones familiares se han caracterizado, por la centralización de las decisiones, baja participación de personal profesional en la gerencia, ausencia de estrategias formales y en general, relaciones limitadas con el entorno, pues su visión de la realidad se circunscribe a clientes, proveedores, 
competidores y en los últimos años, gobierno. Por lo tanto, las decisiones se toman según las condiciones de dicho entorno, actuando de manera reactiva y adaptiva a los eventos externos, en este caso, la función financiera se limita a formular estrategias congruentes con los recursos disponibles y los objetivos esperados, sincronizar flujos monetarios, minimizar costos, establecer controles, que permitan adecuarse a las oportunidades y amenazas del entorno para lograr los objetivos preestablecidos.

Este accionar de las empresas del sector objeto de observación, les ha permitido sobrevivir en los últimos años, pues luego de un crecimiento experimentado durante el periodo 19831999, estas organizaciones han enfrentado una fase de estancamiento, pues sus estrategias reactivas-adaptivas no les ha permitido crecer a partir de 1999, varios son los factores externos que han influido, pues es notorio que enfrentan los efectos de las políticas económicas del Estado, inseguridad vial, ausencia de incentivos para el sector, restricciones para acceder al sistema financiero, factores que inciden negativamente en las decisiones financieras de las pymes, como evidencian los trabajos de Oliveros (2015) y González, Moreno \& Rodríguez (2015) que en el caso de las transportistas, limitan severamente su sostenibilidad y crecimiento.

Igualmente las pymes transportistas enfrentan situaciones de carácter interno, como las capacidades gerenciales y financieras que las sitúan en desventaja frente a competidores externos con mayor capacidad de movilización, costos más bajos y sobre todo capacidad de negociar con el entorno.

Estas restricciones al sistema financiero y debilidades señaladas, parecieran ser una constante en las pymes en otros entornos, pues estudios sobre micros, pequeñas y medianas empresas, realizados en Colombia, coinciden con los planteamientos anteriores, con los hallazgos de Bonilla, Cardeño \& Cardeño, (2015) cuyo estudio sobre la función financiera en las mipymes del municipio Riohacha-Guajira, reveló debilidades en la estructura financiera y limitaciones para acceder al sistema financiero en parte de la población estudiada, igualmente, el trabajo de Pacheco (2013), arrojó limitaciones para la planificación formal en las Pymes de Sincelejo-Sucre, asimismo la investigación de Angulo, Berrio \& Caicedo (2014), reveló debilidades en la posición financiera de las mipymes colombianas del sector textil en el municipio de Maicao-Guajira. 
Estos resultados demuestran que las pequeñas y medianas empresas de diferentes sectores y entornos, presentan dificultades financieras similares y que la gerencia de las mismas requiere herramientas para apoyar la toma de decisiones como los principios de valoración financiera anteriormente descritos.

\section{Metodología}

El proceso metodológico de esta investigación partió del enfoque positivista de la investigación, el estudio es de carácter descriptivo-analítico, cuantitativo y deductivo. La información se obtuvo a través entrevistas semi-estructuradas a los actores involucrados: gerentes, administradores, propietarios. El instrumento de recolección de datos utilizado fue un cuestionario, con 27 ítems, con escala de respuestas, estructurado según la categorización de la variable principios de valoración a través de sus dimensiones, rendimiento, riesgo, valor, liquidez y costo de oportunidad, así como el tipo y objetivo de las técnicas de valoración cuya. La validación se obtuvo mediante la prueba de análisis de contenido y de fuentes documentales.

Los datos suministrados se registraron y se procedió a organizarlos de manera que puedan encontrarse nexos y asociaciones entre los métodos o técnicas de valoración aplicados por estas empresas y los principios de valoración financiera mediante un proceso analítico y reflexivo, propio del método deductivo, tal como señalan Hurtado (2010) y Hernández, Fernández \& Batista (2010). La población estuvo conformada por empresas pequeñas y medianas del sector transporte de carga pesada, inscritas en la Cámara de Transporte del estado Zulia, que tuviesen más de veinte años de operaciones y estén ubicadas en el municipio Maracaibo del mencionado estado. Estos criterios de inclusión de sujetos fueron establecidos con la finalidad de conformar una muestra los más homogénea posible y lograr generar resultados generalizables.

Con la finalidad de contrastar la información suministrada por los informantes del sector, se consultó a un grupo de diez 10 analistas financieros, pertenecientes a instituciones financieras y empresas consultoras en el área, quienes respondieron aspectos vinculados con los principios de valoración; liquidez, valor, rentabilidad y riesgo. 


\section{Resultados}

Los datos aportados por los informantes permitieron alcanzar el objetivo de la investigación referido a analizar los principios de valoración fundamentados en la teoría financiera aplicados por las pequeñas y medianas empresas transportistas de carga pesada, ubicadas en el municipio Maracaibo-Venezuela. Para los efectos, se indagó sobre los métodos y técnicas utilizados para valorar el rendimiento, riesgo, valor, liquidez y costo de oportunidad. En cuanto a los métodos de valoración, gráfico 1, se obtuvo que la gerencia de estas empresas utiliza métodos de valoración del rendimiento que permiten relacionar la utilidad obtenida con la inversión realizada, tales como rentabilidad de los activos, márgenes de rentabilidad, los cuales son de naturaleza estática, por cuanto valoran el resultado para un periodo determinado.

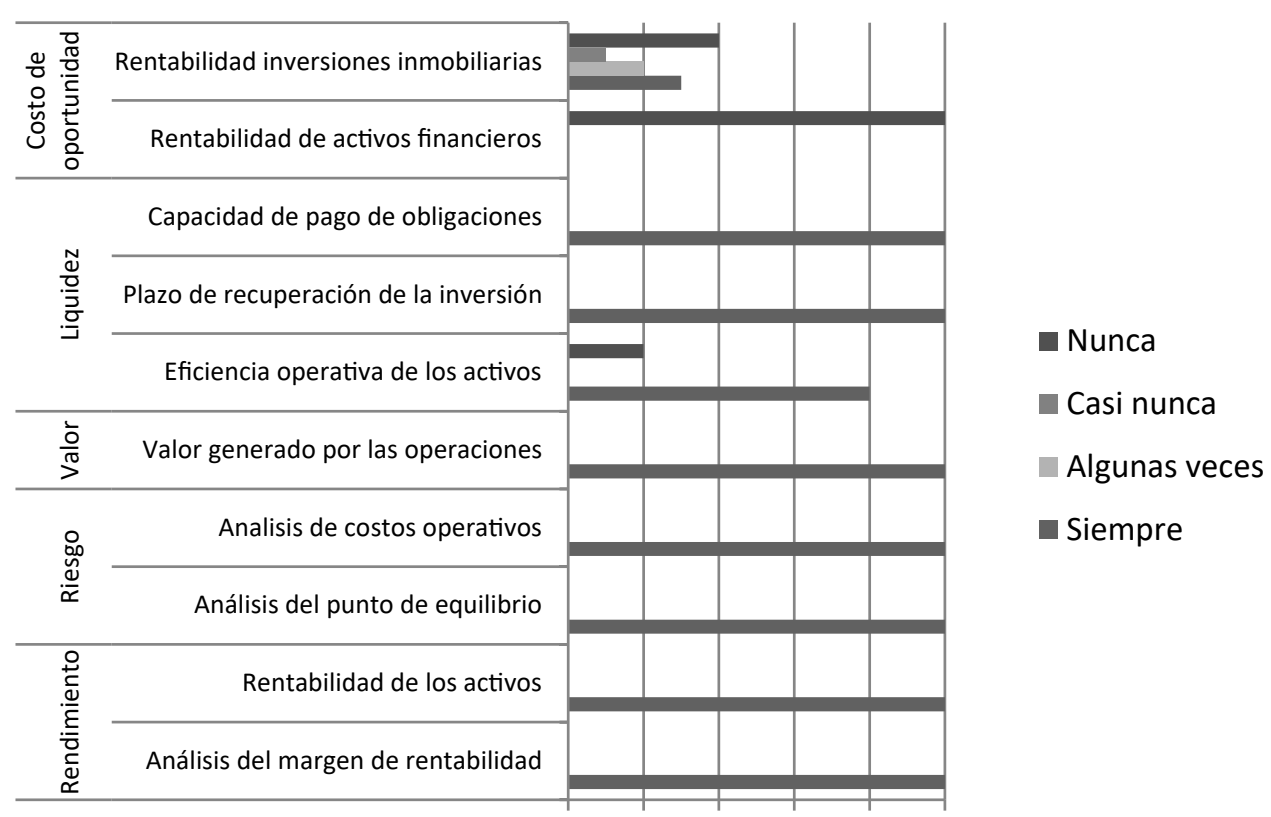

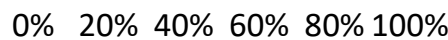

Gráfico 1. Métodos de valoración Elaboración propia (2016), según resultados entrevista 
Esto significa que, si bien estas empresas valoran el rendimiento de sus inversiones, no consideran la variabilidad del valor del dinero en el tiempo, práctica no adecuada según; Mascareñas (2007); Pérez (2009); Eccles (2003), pues relacionan partidas monetarias generadas en diferentes momentos en el tiempo, como por ejemplo, la utilidad neta y el valor de los activos, o la utilidad neta y el patrimonio de los inversionistas, además aún cuando los estados financieros sean ajustados por inflación y reflejen el impacto de la paridad cambiaria, el resultado obtenido es histórico, a una fecha determinada y no considera la actuación futura de la empresa, lo cual resulta inadecuado, más aún en contextos con tendencias hiperinflacionaria, como es el caso venezolano, o entornos dinámicos y complejos (Berk et al., 2010)

Igualmente, la información sobre los métodos para valorar el riesgo indica que son de tipo estáticos, como el análisis de costos y del punto de equilibrio. Esto denota que las empresas están conscientes del riesgo pero la mayoría no utilizan métodos que les permitan valorar el impacto del riesgo en los resultados financieros, tal como indican Gitman \& Zutter (2012).

En cuanto a la medición del valor, utilizan métodos referidos a determinar el valor generado en las operaciones, específicamente utilidad en operaciones y utilidad en ventas, todas ellas de carácter puntual o histórico que impiden valorar el potencial futuro de la empresa, tal como explican Fernández (2002) y Stewart (2000). Los resultados sobre la valoración de la liquidez, demuestran que determinan la capacidad de pago, el plazo de recuperación de la inversión y eficiencia operativa de los activos; estas empresas, si bien consideran importante la posición de liquidez, (Gitman \& Zutter, 2012); utilizan métodos no apropiados para ambientes inflacionarios, como los indicadores de solvencia, rotaciones de activos circulantes y plazo de recuperación de la inversión, pues se comparan cantidades monetarias originadas en fechas o vencimientos diferentes, desconociendo el efecto del valor temporal del capital en la valoración de activos y proyectos. (Ibarra, 2001).

Con relación al costo de oportunidad, la información aportada revela que no utilizan métodos que permitan valorar el costo de oportunidad para tomar decisiones sobre inversiones, por lo tanto desconocen la rentabilidad que pudiera generar una inversión alterna y en consecuencia, no utilizan el referido costo como tasa de descuento para valorar los proyectos de inversión. Garay \& González (2015); Bodie \& Merton (2003) 
Los resultados sobre los métodos de valoración indican que las empresas aplican métodos estáticos apegados a los principios financieros rendimiento, riesgo, valor y liquidez, que les ofrece un resultado puntual para un momento determinado y no les permite valorar las capacidades o potencialidades de la empresa para generar valor, lo cual contradice los principios del valor temporal del capital y costo de oportunidad, sobre el cual descansan las técnicas dinámicas de valoración empleadas en ambientes inflacionarios. (Gitman \& Zutter, 2012); (Berk et al., 2010); (Mascareñas, 2007); (Pérez, 2009).

De acuerdo con las técnicas de valoración utilizadas por las pyme transportistas, ver gráfico 2 , los resultados obtenidos demuestran que aplican técnicas de naturaleza mixta, combinando elementos cuantitativos y cualitativos que permitan evaluar el desempeño financiero.

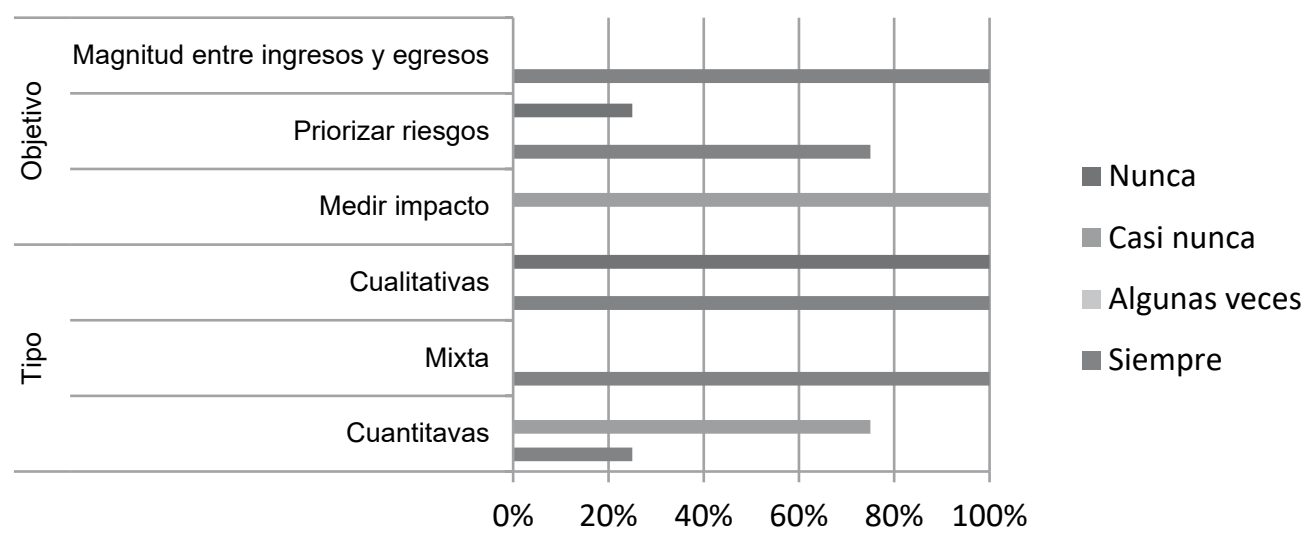

Gráfico 2. Técnicas de valoración

Elaboración propia (2016), según resultados entrevista.

En función del objetivo relacionado con las técnicas aplicadas, coincidieron en señalar que buscan determinar la magnitud entre ingresos y egresos, es decir el punto de equilibrio, destaca que las técnicas de valoración aplicadas no miden el impacto del riesgo y por lo tanto toman decisiones sin considerar los efectos de eventos futuros sobre los resultados de sus inversiones. 
Esta práctica puede conducir a elegir alternativas de inversión riesgosas o que en el corto plazo contribuyan con la destrucción del valor. (Bodie \& Merton, 2003); (Garay \& González, 2015)), (Berk et al., 2010). Otro aspecto indagado fue la aplicación de los principios de valoración financiera por parte de los responsables de la generación de información y toma de decisiones, los resultados se muestran en el gráfico 3, como puede observarse, los entrevistados indicaron que siempre utilizan los principios de rendimiento y liquidez, los otros principios no son aplicados por la mayoría, pues indicaron que siempre evalúan el riesgo (30\%), la creación de valor (25\%) y el costo de oportunidad (10\%).

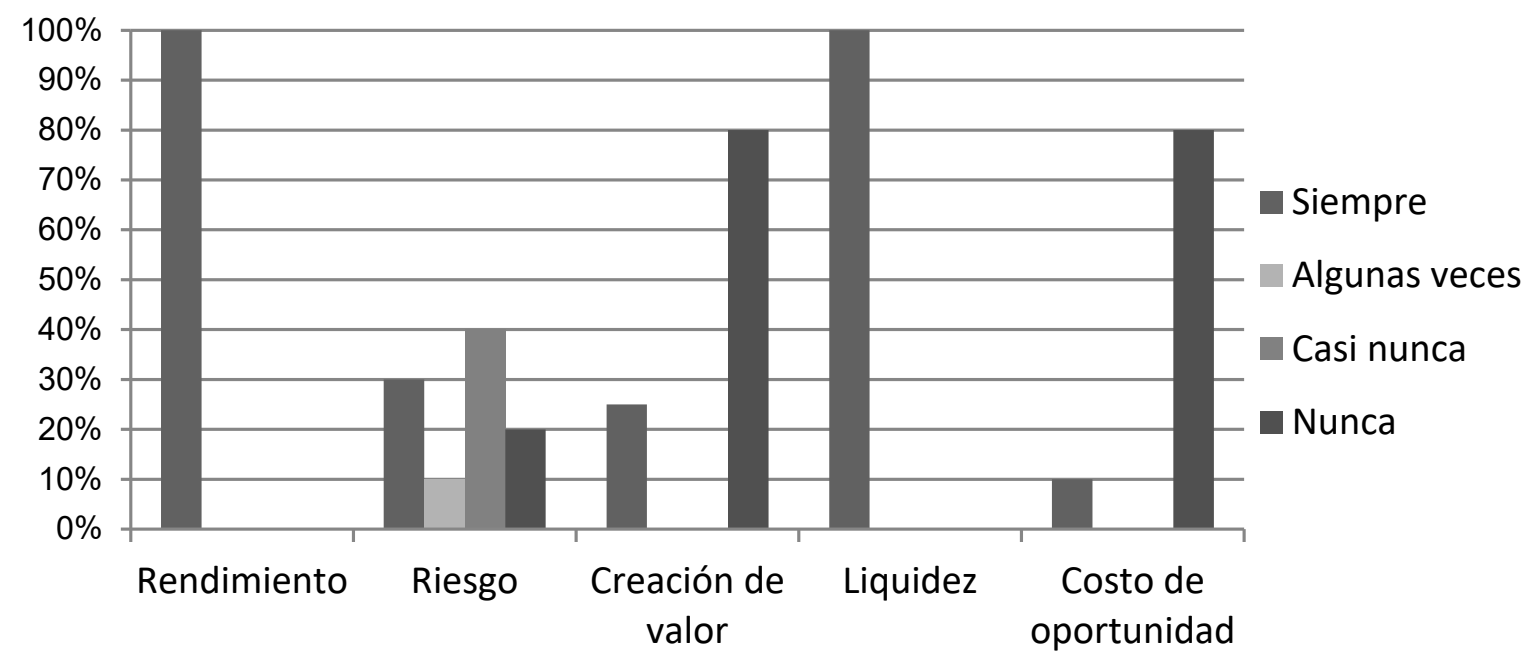

Gráfico 3. Aplicación de los principios de valoración

Elaboración propia (2016), según resultados entrevista

Significa entonces, que la mayoría de los principios de valoración no son aplicados por estas empresas, debido en parte al desconocimiento expresado sobre las mismas, la calidad de la información financiera generada y la falta de una política financiera que oriente la toma de decisiones sobre este particular. 
La información aportada por los analistas financieros para valorar el desempeño de las pymes transportistas, reveló diferencias de criterios entre los informantes de las empresas y los mencionados analistas, pues como se evidencia en el gráfico 4, los responsables del análisis para el otorgamiento de crédito y consultores gerenciales aplican criterios y técnicas de carácter dinámico fundamentados en los principios financieros ya referidos.

La mayoría consultada expresó que, siempre valoran la capacidad de generación de fondos futura (100\%), la creación de valor (90\%), el descuento de flujos de fondos (100\%), tasa interna de rendimiento (90\%), análisis de escenarios (100\%) y simulación (80\%). En cuanto a los criterios aplican la rentabilidad, el riesgo, costo de oportunidad, liquidez y consideran el valor temporal del dinero.

Esto significa que las valoraciones de los analistas financieros proporcionan una evaluación más objetiva y coherente con las condiciones del entorno venezolano, lo cual difiere de las estimaciones realizadas por las empresas observadas.

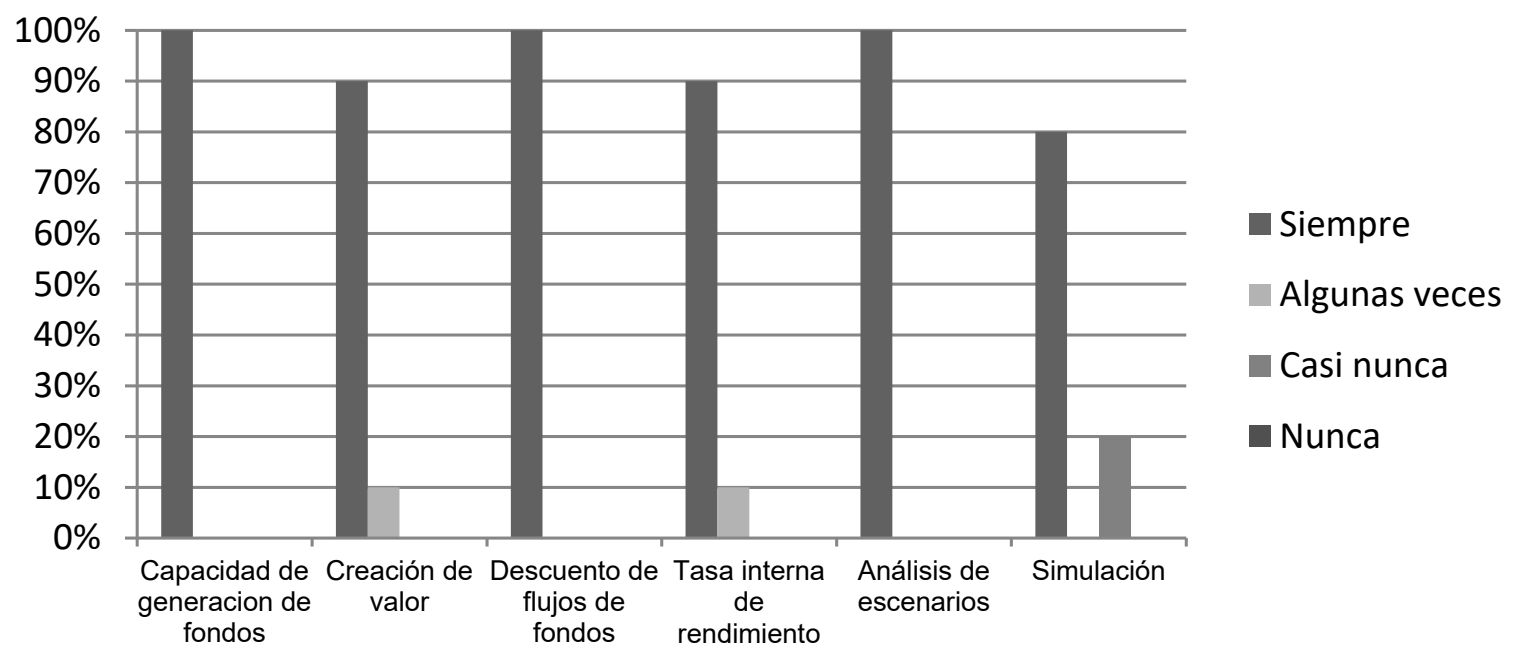

Gráfico 4. Criterios y técnicas aplicados por los analistas financieros Elaboración propia (2016), según resultados entrevista 
Otro aspecto destacado por los consultores gerenciales se refirió a la calidad de la información financiera, pues al momento de realizar sus análisis, requieren información financiera no disponible, lo cual los obliga a colectar, organizar y generar los datos necesarios para realizar sus estudios y reportes.

\section{Conclusiones}

El analizar los principios de valoración económica fundamentados en la teoría financiera aplicados en las pequeñas y medianas empresas Pyme de Maracaibo- Venezuela, permitió concluir sobre los principios de valoración fundamentados en la teoría financiera aplicados en las pymes transportistas de carga pesada del Municipio Maracaibo permitió establecer lo siguiente:

- Los métodos de valoración utilizados son de carácter estático y generan valoraciones puntuales que no informan suficientemente sobre las potencialidades económicas-financieras de las empresas.

- Las técnicas de valoración de riesgo empleadas no analizan el impacto de eventos futuros no deseados sobre los resultados económicos de las empresas.

- Los principios de valoración financiera son aplicados parcialmente, solo enfatizan rendimiento y liquidez, desconociendo los beneficios de valorar el riesgo, la capacidad de generación de valor y costo de oportunidad.

- Los criterios y técnicas utilizados por los analistas para valorar el desempeño financiero involucran aspectos no considerados por las empresas observadas y que están referidos a la generación de valor, valor temporal del dinero y costo de oportunidad.

- En consecuencia se observaron diferencias entre las valoraciones realizadas por las empresas y los analistas-consultores, explicado en parte por la calidad de la información. Igualmente, los analistas de crédito de las instituciones financieras enfrentan dificultades para realizar sus estudios del perfil crediticio de las empresas debido a la asimetría de la información. 
- La valoración inadecuada por parte de los responsables de la función financiera, explica en parte, los resultados obtenidos en los últimos diez años, donde han visto disminuir su rentabilidad, capacidad de crecimiento y sostenimiento de manera substancial, al punto que han cesado en operaciones o redimensionado su tamaño.

- La valoración financiera en estas empresas debe considerar los elementos aportados por los analistas-consultores a fin de lograr evaluaciones integrales que consideren las potencialidades de las empresas, su entorno y los principios financieros.

- Estas valoraciones permitirán diagnosticar la empresa, analizar sus capacidades financieras, estudiar las fuerzas del entorno y su influencia en el desempeño de la empresa, así como criterios para seleccionar alternativas de inversión y financiamiento considerando el rendimiento, el riesgo, la liquidez, el valor temporal del dinero y el costo de oportunidad.

\section{Referencias}

Angulo, F. A., Berrío, H. J. \& Caicedo, L. (2014). Estrategias de inversión en capital de trabajo aplicadas por las micros, pequeñas y medianas empresas colombianas de comercio textil en el municipio de Maicao. Revista Dimensión Empresarial, vol. 12, núm. 2. p. 69-82.

Bonilla, E., Cardeño, E. \& Cardeño N. (2015) La función financiera en las micro, pequeñas y medianas empresas del municipio de Riohacha. Económicas CUC, 36(2) 137-146

Berk, J.; DeMarzo P. \& Harford, J. (2010). Fundamentos de Finanzas Corporativas. Madrid-España. Pearson Educación, S.A.

Bodie, Z.; Kane, A. \& Marcus, A. (2004). Principios de Inversiones. Quinta Edición. Madrid, España. McGraww Hill Interamericana de España, S.A.U.

Bodie Z. \& Merton R. (2003) Finanzas. Edición revisada. Pearson Educación. México.

Copeland, T.; Koller, T. \& Murrin, J. (2004) Valoración. Medición y Gestión del Valor. Barcelona, España. Ediciones Deusto. 
Eccles, R. (2003) Manifiesto sobre la medida del rendimiento. Harvard Business Review. Cómo medir el rendimiento de la empresa. Barcelona., España. Planeta DeAgostini Profesional y Formación S.L.

Fernández P. (2002) Valoración de empresas. 2da. Edición. Barcelona, España. Ediciones Gestión 2000, S.A.

Garay U. \& González M., (2015), Fundamentos de Finanzas con aplicaciones al mercado venezolano. 2da. Edición. Caracas, Venezuela. Ediciones IESA

Gitman, L. \& Zutter, Ch. (2012) Principios de administración financiera Decimosegunda edición. México. Pearson Educación.

González, J.; Moreno, L. \& Rodríguez, M. (2015). La integración y sus convenios internacionales en América Latina. Económicas CUC, 36(2) 79-94

Hernández, R., Fernández, C. \& Baptista P. (2014) Metodología de la investigación. 6ta. Edición. México. McGraw Hill/Interamericana Editores, S.A. de C.V.

Hurtado de Barrera, J. (2010). Metodología de la Investigación Holística Cuarta Edición. Caracas, Venezuela. Quirón Ediciones.

Ibarra, Alberto. (2001). Análisis de las dificultades financieras de las empresas en una economía emergente: las bases de datos y las variables independientes en el sector hotelero de la Bolsa Mexicana de Valores. Barcelona - España. Tesis Doctoral. Departamento de Economía de la Empresa. Universidad Autónoma de Barcelona. Disponible en: http:// www.eumed.net/tesis-doctorales/2010/aim/factor\%20de\%20liquidez.htm. Fecha de consulta: $12 / 01 / 2016$

Kantis, Hugo (2004) Desarrollo emprendedor: América Latina y la experiencia internacional. Estados Unidos. Banco Interamericano de Desarrollo.

Mascareñas Juan (2007) Principios de Finanzas. Madrid-España. Universidad Complutense de Madrid.

Oliveros, J. (2015). Estructura económico-financiera de pequeñas y medianas empresas manufactureras colombo-venezolanas. Revista Venezolana de Gerencia (RVG). Año 20. N 71. Universidad del Zulia (LUZ). pp 397- 418 
Pérez J. (2009). Principios y teorías de las Finanzas en las inversiones financieras. Caracas, Venezuela. Liven Editores, C.A

Pacheco, C. (2013). Estrategias empresariales más utilizadas por las pymes en Sincelejo. Revista Económicas CUC, Vol. 34, Nº 1, pp. 183-202. Barranquilla, Colombia. Editorial Educosta.

Stewart, B. (2000). En busca del Valor. Barcelona, España. Ediciones 2000. 